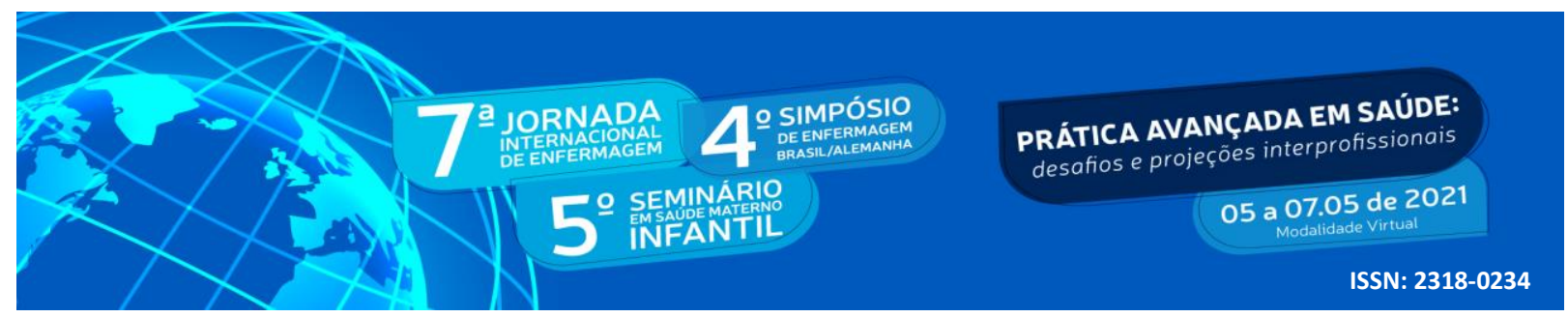

DOI: http://doi.org/10.48195/jie2021-047

\title{
POTENCIALIDADES E FRAGILIDADES DO ENVELHECIMENTO NO MEIO RURAL: REVISÃO NARRATIVA ${ }^{1}$
}

\author{
Eduardo Marques Machado²; Claudia Maria Ferrony Rivas ${ }^{3}$; Karol Tolfo ${ }^{4}$; Natanna \\ Rosa $^{5}$ Maria Helena Gehlen; ${ }^{6}$ Naiana Oliveira dos Santos ${ }^{7}$
}

\begin{abstract}
RESUMO
O presente trabalho objetiva conhecer a literatura científica acerca das fragilidades e potencialidades do processo de envelhecimento no meio rural. A metodologia utilizada foi um estudo de revisão narrativa de literatura. A pesquisa bibliográfica desenvolvida na base de dados lilacs e google scholar. Para conduzir a revisão de narrativa, elencou-se a seguinte questão norteadora da pesquisa: $\mathrm{O}$ que se tem produzido na literatura científica acerca das fragilidades e potencialidades do processo de envelhecimento no meio rural. Tendo como resultados e discussões duas categorias dos estudos analisados, sendo elas: Potencialidades do envelhecimento em zona rural; Fragilidades do envelhecimento em zona rural. Sendo discorridas a partir das perspectivas do envelhecimento rural. Conclui-se que com as potencialidades e fragilidades, é possível realizar maiores discussões referentes a políticas públicas de saúde, com o objetivo de realizar a promoção da saúde. Cabe ressaltar, que são necessárias maiores discussões e pesquisas acerca da temática.
\end{abstract}

Palavras-chave: Envelhecimento; Enfermagem; Qualidade de Vida.

\begin{abstract}
The present work aims to know the scientific literature about the weaknesses and potentialities of the aging process in rural areas. The methodology used was a study of narrative literature review. The bibliographic research developed in the lilacs and google scholar database. To conduct the narrative review, the following guiding question of the research was listed: What has been produced in the scientific literature about the weaknesses and potentialities of the aging process in rural areas. Having as results and discussions two categories of studies analyzed, namely: Potentialities of aging in rural areas; Weaknesses of aging in rural areas. Being discussed from the perspective of rural aging. It is concluded that with the potentialities and weaknesses, it is possible to carry out further discussions regarding public health policies, with the aim of promoting health. It should be noted that further discussions and research on the subject are needed.
\end{abstract}

\footnotetext{
1 Trabalho de pesquisa do Núcleo de Ensino, Extensão e Pesquisa do Processo de Envelhecimento

2 Estudante do Curso de Enfermagem. Universidade Franciscana. E-mail: eduardomarques051@gmail.com

3 Estudante do Curso de Enfermagem. Universidade Franciscana. E-mail: claudiamfrivas@gmail.com

4 Estudante do Curso de Enfermagem. Universidade Franciscana. E-mail: karoltolfo98@ gmail.com

5 Enfermeira. Especialista em Oncologia. E-mail: natannarosa0509@gmail.com

6 Orientadora. Doutora. Professora do Curso de Enfermagem. Universidade Franciscana. E-mail:

mah@ufn.edu.br

7 Orientadora. Doutora. Professora do Curso de Enfermagem. Universidade Franciscana. E-mail: naiana.santos@ufn.edu.br
} 


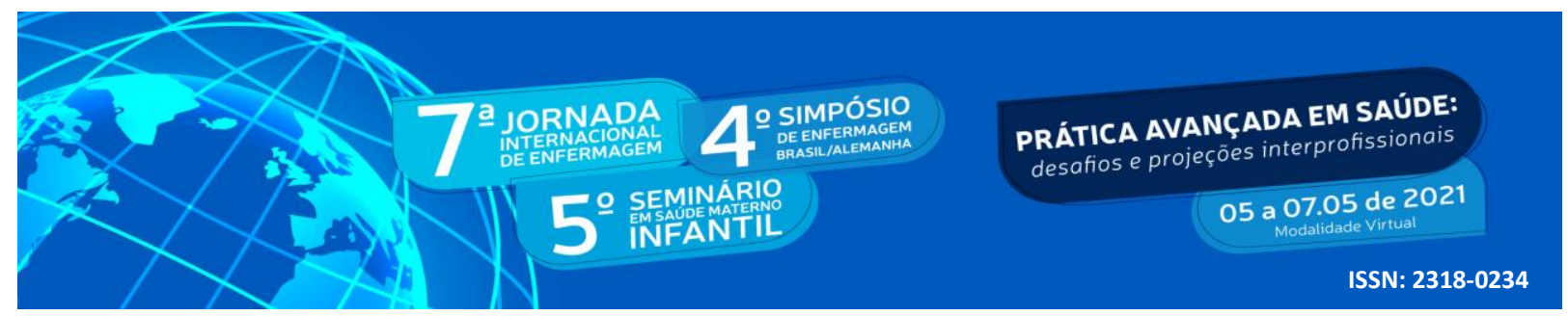

Key Words: Aging; Nursing; quality of life.

\section{INTRODUÇÃO}

O envelhecimento da sociedade é uma realidade mundial que tem ocorrido de modo diferenciado em diversos países, sendo de grande responsabilidade da saúde pública (PAIVA; HILLESHEIM; HAAS, 2019). Com as diferenças sociais, cabe visualizar como está ocorrendo esse processo em zonas rurais e populações do campo. Visto que diferentes problemáticas atingem esses indivíduos.

Envelhecer na zona rural apresenta algumas singularidades, representadas em um estilo de vida saudável e atividades cotidianas ativas. Assim, o estilo de vida saudável se apresenta pelo fato de produzir o próprio alimento e maior ingestão de frutas, verduras e legumes. Porém, também se visualiza maior ingesta de gorduras e doces. Quanto às atividades cotidianas, se tratam do trabalho físico como cuidar de áreas de produção agropecuária (LLANO et al., 2017).

Os fatores citados anteriormente se referem desde a concepção cultural desses indivíduos, onde o idoso considera o seu trabalho junto ao campo como uma atividade de lazer. Visto que em diversas oportunidades os mesmos se sentem incomodados no meio urbano. Assim, a zona rural gera qualidade de vida e segurança a aquele indivíduo.

A promoção da saúde integral e bem-estar na velhice passa pela oferta de autonomia e independência. Essas se tornam um desafio em zonas rurais pelo fato de que o acesso aos bens e serviços são dificultados, principalmente pela distribuição geográfica (GRDEN et al., 2019). Desta forma, cabe aos agentes públicos, formularem políticas e ações visando essa população, para a promoção da qualidade de vida e do envelhecimento saudável em todos os ambientes.

O envelhecimento no território brasileiro trata-se de um processo heterogêneo, onde o ambiente rural apresenta singularidades (KONZEN; FREITAS, 2019). Se faz necessário a compreensão dos diferentes aspectos do processo de envelhecimento, sendo um deles o envelhecimento na zona rural.

\section{OBJETIVO}




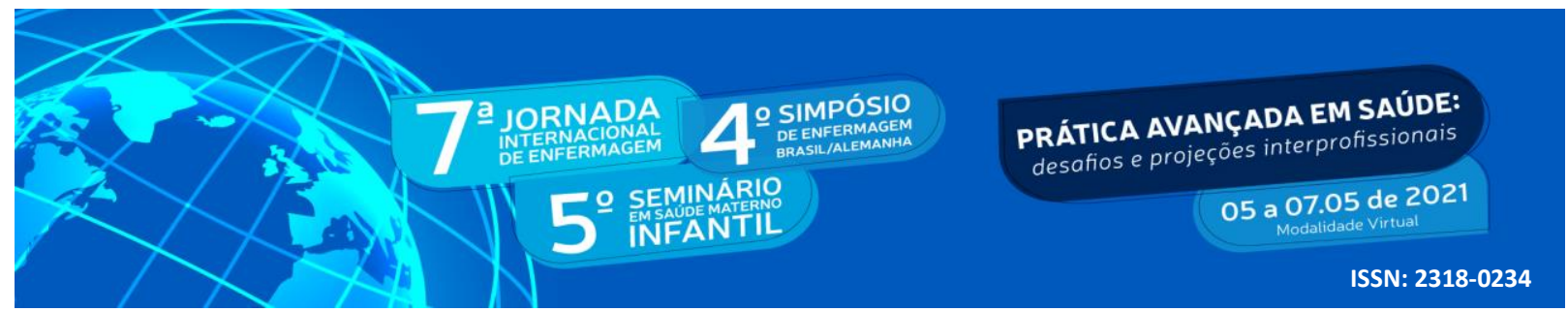

Conhecer a literatura científica acerca das fragilidades e potencialidades do processo de envelhecimento no meio rural.

\section{METODOLOGIA}

Trata-se de um estudo de revisão narrativa de literatura. A pesquisa bibliográfica foi desenvolvida na base de dados, Literatura Latino-Americana e do Caribe em Ciências da Saúde (LILACS) e Google Scholar.

A busca das referências ocorreu durante o mês de janeiro a março de 2021. Foram utilizados como descritores : envelhecimento; zona rural; qualidade de vida. Para conduzir a revisão de narrativa, elencou-se a seguinte questão norteadora da pesquisa: O que se tem produzido na literatura científica acerca das fragilidades e potencialidades do processo de envelhecimento no meio rural.

Quanto aos critérios de inclusão considerou-se: os artigos completos disponíveis em português que abordassem a temática com recorte temporal de quatro anos, pelo fato de obter resultados atualizados. Os critérios de exclusão foram artigos que não estivessem disponíveis na íntegra, teses, dissertações e os que não atendessem a questão de pesquisa.

Nos resultados referentes às bases de dados foram achados três artigos na base de dados Lilacs e selecionado um. No Google Scholar foram achados 16 e selecionados seis. A análise dos dados ocorreu na forma descritiva. Sendo que compoem os resultados com três artigos referentes as potencialidades e cinco correspondem as fragilidades, sendo que desses dois artigos abordam potencialidades, portando utilizados em ambas categorias.

\section{RESULTADOS E DISCUSSÃO}

Diante da analise dos artigos selecionados, optou-se pela divisão em duas subcategorias, sendo: Potencialidades do envelhecimento em zona rural e Fragilidades dos envelhecimento em zona rural. 


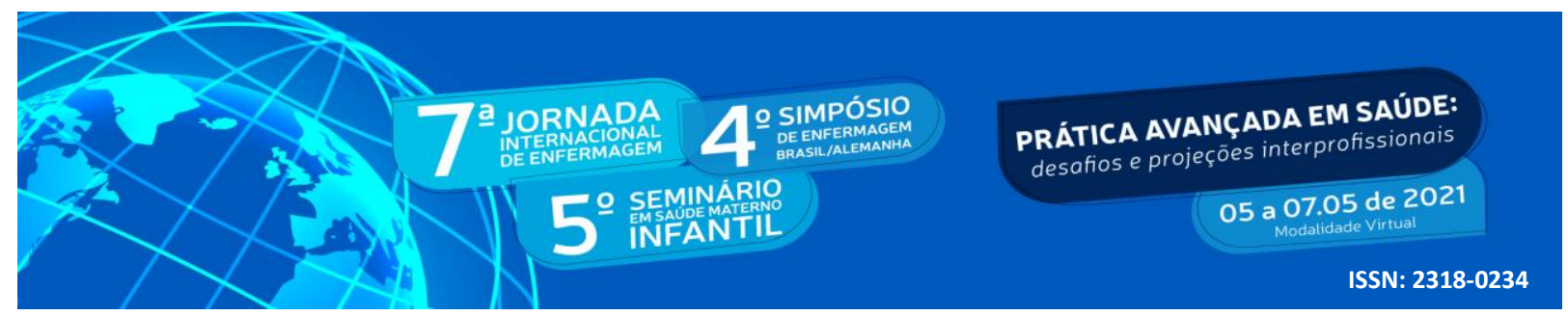

\subsection{Potencialidades do envelhecimento em zona rural}

Em uma pesquisa realizada com idosos comunidade do Arisco do Sutero, localizada em região de clima semiárido do Rio Grande do Norte. Participaram da pesquisa idosos aposentados (mulhres 55 anos e homens 60 anos de idade), residentes da comunidade. $\mathrm{O}$ envelhecimento no ambiente rural carrega perspectivas positivas para o desenvolvimento desse processo, como o contato com a natureza e a possibilidade de viver dela, se alimentar da mesma, sem o uso de agentes químicos. A possibilidade de produzir o próprio alimento, da forma que o indivíduo pretender e o mais importante desse processo se trata de realizar isso em família, estar muitas vezes em contato com os filhos e participar da criação dos netos (COSTA; COSTA; DANTAS, 2019).

Envelhecer no meio rural proporciona com que os idosos estejam imersos em seu cotidiano. Assim, se mostram ativos, realizando trabalho braçal e manual, tendo relação direta com sua funcionalidade, proporcionando autonomia e independência (COSTA; LEÃO; CAMPOS, 2020).

Viver a velhice no ambiente rural implica em uma realidade de maior segurança, maior qualidade de vida e melhores relações de reciprocidade e solidariedade do que no meio urbano (PEREIRA et al., 2020). Essas perspectivas estão sendo reduzidas no meio urbano, devido a fatores como alto estresse diário, grandes jornadas de trabalho e o uso indiscriminado de tecnologias nas relações interpessoais. Levando em consideração aos idosos esses fatores foram sendo inseridos ao longo do tempo, principalmente devido a transição demografica, sendo que não faziam parte de seu cotidiano.

As potencialidades descritas colaboram para a promoção da saúde. Observando as mesmas, se pode fazer uma relação com determinantes sociais de saúde, como as condições de vida e trabalho; redes sociais e comunitarias; condições socioeconomicas, culturais e ambientais gerais. Onde esses estão aplicados ao cotidiano do idoso, observando o contexto social. 


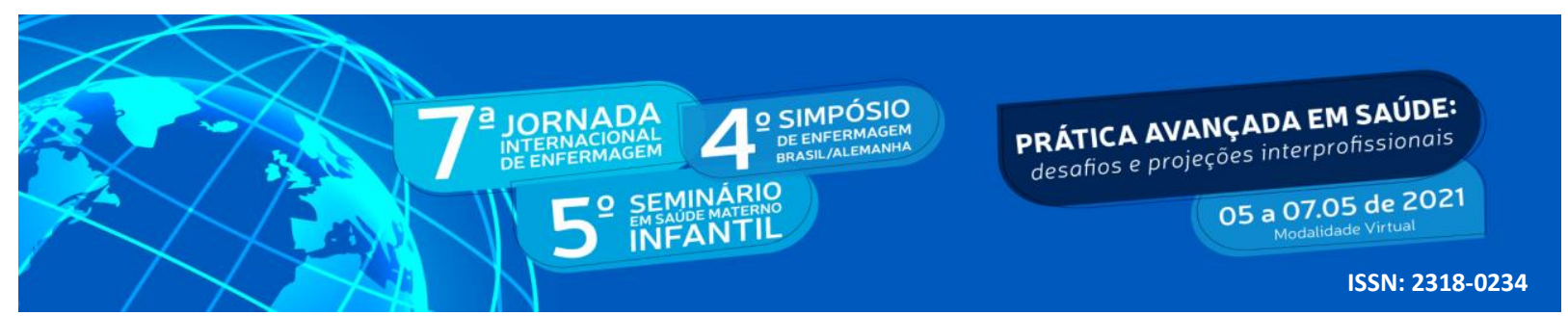

\subsection{Fragilidades do envelhecimento em zona rural}

Os idosos residentes no meio rural, apresentam menor índice de escolaridade. Esse fato acontece devido a educação não ser prioridade, o idoso advém de uma cultura onde o trabalho na roça e a subsistência econômica são mais importantes (COSTA; LEÃO; CAMPOS, 2020).

Os idosos residentes no meio rural apresentam menor nível socioeconômico, podendo se relacionar com a falta de escolaridade, o estudo mostra que mais de $47 \%$ dos idosos são analfabetos. Quanto ao nivel socioeconomico, mais de 57\%, estão na classe D e E. Os idosos de áreas rurais também utilizam em menor escala os serviços privados de saúde, também pode haver relação com a classe econômica, conforme o mesmo estudo apenas 9,5\% dos participantes tem acesso a planos privados de saúde (BÓS et al., 2018).

Ao se pensar na sociedade de zonas rurais, muitas famílias não têm um trabalho fixo com renda fixa. As condições econômicas, são resultado da produção e comercialização do que o campo lhes proporciona, principalmente alimentos, muitos advêm do sistema da agricultura familiar. Com isso, o resultado econômico depende das condições climáticas, por exemplo.

A renda das pessoas idosas que residem em meio rural em grande maioria é resultados do beneficio de aposentadoria ou de uma pequena produção comercial. Porém, atualmente os idosos que envelhecem no meio rural, com essas fontes de renda, apontam para o disparador de uma realidade onde predomina a pobreza, isolamento, baixos níveis educacionais, residências mais precárias, limitações de transporte, problemas crônicos de saúde e distância dos recursos sociais e de saúde (MORAIS; RODRIGUES; GERHARDT, 2008).

As situações evidenciadas anteriormente tem relações diretas com as fragilidades no processo de envelhecimento, uma vez que pela falta de estudo e condições socioeconomicas os idosos encontran-se em uma menor distancia da vulnerabilidade social. Ainda, pode representar uma dificuldade de ir até o serviço, pelas condições sociais e de se sentir em um estado de menosprezo pelo fato da falta de escolaridade.

Existe uma dificuldade no acesso aos serviços de saúde por essa população, representadas por longas distâncias até um serviço de emergência, indicando uma vulnerabilidade e uma condição a ser observada (PEREIRA et al., 2020). 


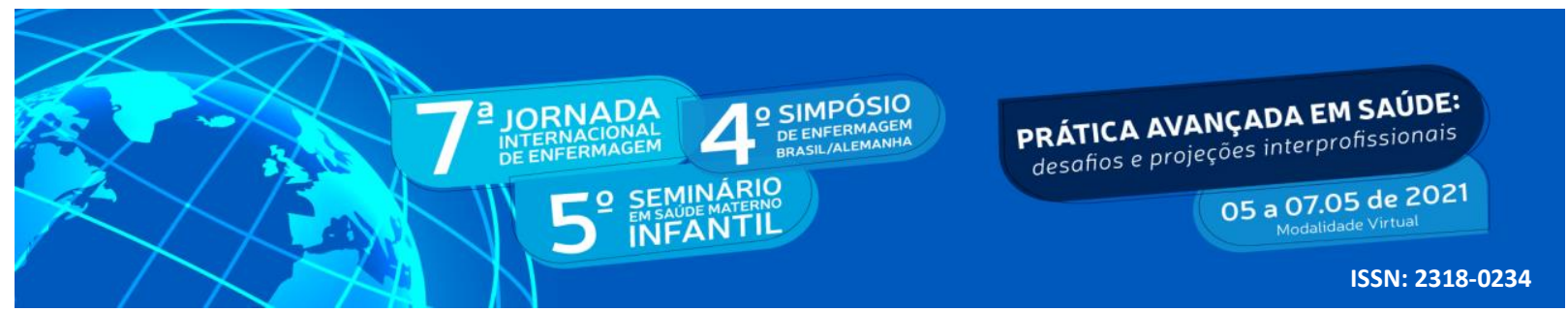

A dificuldade no acesso aos serviços de saúde, se trata de uma problemática que exige resolutividade principalmente dos serviços de saúde e infraestrutura. A acessibilidade à saúde para as pessoas idosas deve ser prioridade, visto o risco de vulnerabilidade e por se tratar de uma população que tem prioridade no acesso à saúde. Durante a busca na literatura, não apareceram estudos que mostrem como ocorre o atendimento de urgencia e emergencia a idosos residentes em meio rural. Unidades de saúde em zonas rurais tambem não foram descritas. Os idosos residentes em zonas rurais não podem ser esquecidos, se faz necessário a criação de ações eficazes para a promoção integral a saúde da pessoa idosa.

Trabalhadores rurais, incluindo os idosos, em determinadas situações de seu trabalho estão expostos a intoxicações por agrotóxicos, essa situação ocorreu em um dos estudos utilizados. Esse fato ocorre devido ao uso incorreto dos Equipamentos de Proteção Individual (EPIs), a manipulação de agrotóxicos em determinadas situações ocorre por indivíduos não alfabetizados, potencializando o risco (CORCINO et al., 2019). Uma forma de resolver essa problemática se trata da educação em saúde para quem tem contato com os agentes químicos, visto que esses causam danos à saúde.

Diante do exposto, como em todos os ambientes o meio rural possui fragilidades, essas podem potencializar o processo de envelhecimento e ao mesmo tempo reduzir a qualidade de vida. Para que isso não torne-se uma problemática, se faz necessário ações de saúde pública e promover a atenção integral à pessoa idosa em todos os contextos.

\section{CONCLUSÃO}

Conhecer os fatores que influenciam no processo de envelhecimento na zona rural, a saúde e como estes se apresentam no cotidiano colabora diretamente na compreensão da qualidade de vida dos idosos nesse cenário. Assim, proporcionando um envelhecimento ativo e independente.

O envelhecimento no contexto rural possui potencialidades e fragilidades. Como perspectivas positivas, foi possivel identificar alimentação saudavel, maior vinculo familiar, menor estresse, maior segurança e promoção da funcionalidade e autonomia. Referente a fragilidades, foi notorio, menor indice de escolaridade, menor nivel socioeconomico, 


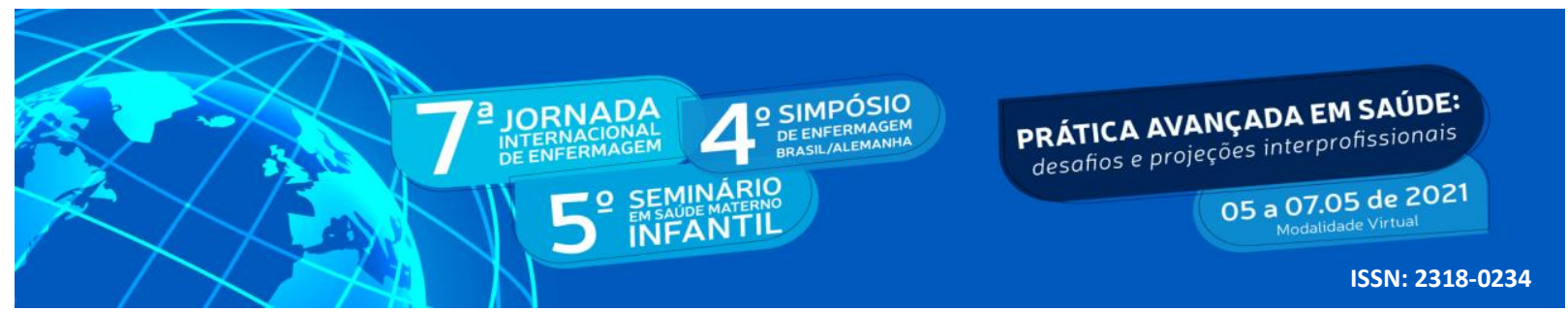

dificuldade no acesso aos serviços de saúde e exposição a agrotoxicos na realização de seu trabalho.

O presente trabalho evidencia situações de fragilidade e situações com potencialidades, referentes ao envelhecimento no ambiente rural. Com isso, fica possível realizar maiores discussões referentes a políticas públicas de saúde, com o objetivo de realizar a promoção à saúde. Cabe pontuar, que são necessárias maiores discussões e pesquisas acerca da temática. Também vale ressaltar que com maiores estudos acerca da temática, serão evidenciadas novas potencialidades e fragilidades.

\section{REFERÊNCIAS}

BÓS, A. J. G. et al. Diferenças no perfil socioeconômico e de saúde de idosos do meio rural e urbano: pesquisa nacional de saúde, 2013. Geriatr Gerontol Aging., vol. 12, n. 3, p. 148-153, Rio de Janeiro, 2018.

COSTA, J; COSTA, L; DANTAS, C. Pessoas idosas e sentidos de rural no interior do Rio Grande do Norte. Rev. Polis e Psique, vol. 10, n. 1, p. 164 - 186, Porto Alegre, 2019.

COSTA, R. S. DA.; LEÃO, L. F.; CAMPOS, H. L. M. Envelhecer na zona rural do interior do estado do Amazonas, desempenho cognitivo, funcionalidade e percepção de saúde: um estudo transversal. Revista Kairós-Gerontologia, vol. 23, n. 1, p. 83-103, São Paulo, 2020.

CORCINO, C. O. et al. Avaliação do efeito do uso de agrotóxicos sobre a saúde de trabalhadores rurais da fruticultura irrigada. Ciênc. saúde coletiva, vol. 24, n. 8, p. 3117-3128, Rio de Janeiro, 2019.

MORAIS, E. P.; RODRIGUES, R. A. P.; GERHARDT, T. E. Os idosos mais velhos no meio rural: realidade de vida e saúde de uma população do interior gaúcho. Texto Contexto Enferm, vol. 17, n. 2, p. 374-83, Florianópolis, 2008

GRDEN, C. R. B. Atuação da residência multidisciplinar em saúde do idoso em uma comunidade rural. Revista Conexão, vol. 15, n. 2, p. 187-192, Caxias Do Sul, 2019.

KONZEN, L. T.; FREITAS, C. D. R. ACESSO À SAÚDE POR IDOSOS RURAIS. XVI Seminário Internacional Demandas Sociais e Políticas Públicas na Sociedade Contemporânea. Universidade de Santa Cruz Do Sul, Santa Cruz do Sul, 2019.

LLANO, P. M. P. et al. Fragilidade em idosos da zona rural: proposta de algoritmo de cuidados. Acta paul. enferm. vol.30, n.5, p. 520-530, São Paulo, 2017. 


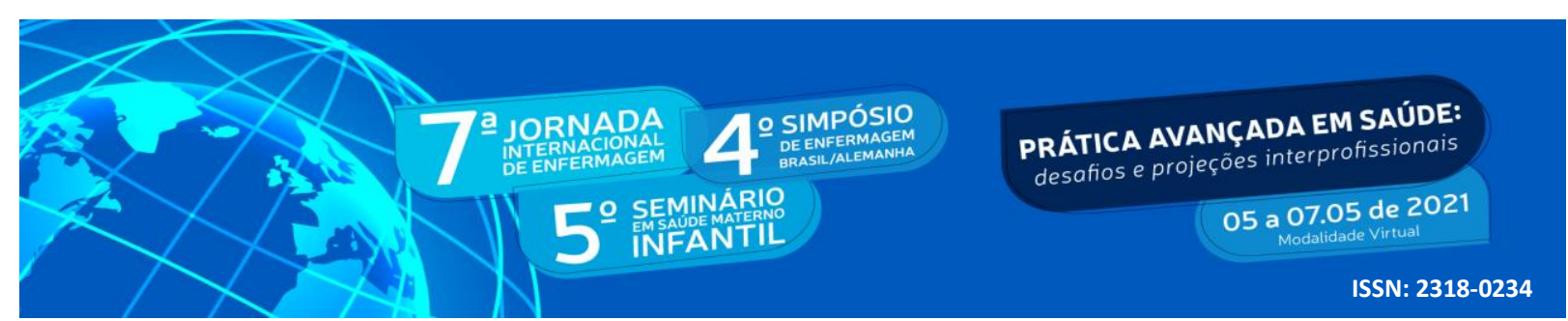

PAIVA, K. M.; HILLESHEIM, D.; HAAS, P. Atenção ao idoso: percepções e práticas dos Agentes Comunitários de Saúde em uma capital do sul do Brasil. CoDAS, vol. 31, n. 1, e20180069, São Paulo, 2019.

PEREIRA, J. S. et al. Representações sociais acerca do processo de envelhecimento no meio rural. Revista Kairós-Gerontologia, vol. 23, n. 2, p. 457-475. São Paulo, 2020. 